\title{
Production spermatique et fertilité de verrats soumis à un rythme intensif d'éjaculation
}

\author{
R. NOWAK, M. PAQUIGNON *, J.P. SIGNORET \\ avec la collaboration technique de J. Gautier, D. Col et P. Despres \\ I.N.R.A., Physiologie de la Reproduction, F 37380 Nouzilly \\ * I.T.P., 149, rue de Bercy, F 75595 Paris Cedex XII
}

\begin{abstract}
Résumé
Nous avons examiné la production de sperme chez le verrat soumis à un rythme intensif de collecte puis nous avons comparé cette situation avec celle de l'accouplement naturel et enfin, nous avons testé la fertilité des truies après accouplement naturel sous différents rythmes d'éjaculation.

Chaque verrat a été collecté à raison de 1,2 et 4 éjaculats par jour pendant 5 jours avec une période de repos sexuel de 2 semaines entre chaque série d'éjaculations. Pour valider les résultats en accouplement naturel, des truies ont été abattues immédiatement après la saillie afin de mesurer la production de sperme déposé dans l'utérus. La fertilité a été vérifiée après abattage de truics 30-35 jours après la saillie.

Pour le rythme d'une fois par jour, la production de sperme par éjaculat décroît linéairement du premier au cinquième jour de récolte. Pour les rythmes 2 et 4 , la chute de production du premier jour est suivie par une production spermatique plus régulière qui, dès le troisième jour, n'excède pas $10 \times 10^{9} \mathrm{spz}$ pour le rythme 2 et dépasse rarement $5 \times 10^{9}$ pour le rythme 4. L'augmentation de la fréquence des collectes a pour effet de réduire considérablement lc nombre de spermatozoïdes par éjaculat : 12 p. 100 et 31 p. 100 des éjaculats respectivement aux rythmes 2 et 4 contiennent moins de $3 \times 10^{9} \mathrm{spz}$.

Quel que soit le rythme d'éjaculation utilisé, il n'y a pas de différence significative entre la collecte et l'accouplement en ce qui concerne le volume de l'éjaculat aussi bien que le nombre de spermatozoïdes émis. Nous avons constaté, durant les saillies, un important reflux qui atteint 40 p. 100 de l'éjaculat et concerne autant le volume que le nombre de spermatozoïdes.

Dans nos conditions expérimentales, la tertilité décroît quand le rythme d'éjaculation est le plus élevé : 91,6 p. 100 contre 58,3 p. 100 respectivement pour une et quatre éjaculations par jour. Ceci pourrait être dû à un trop faible nombre de spermatozoïdes mis en place lors de l'accouplement lorsque le mâle est soumis à un rythme d'éjaculation intensif.
\end{abstract}

Mots clés : Verrat, spermatozoïdes, rythme de collecte, saillie, fertilité.

\section{Introduction}

La production spermatique est un phénomène continu. A la sortie des tuber séminifères, les spermatozoïdes transitent dans l'épididyme où ils subissent les transformations nécessaires à l'apparition de leur pouvoir fécondant (lapin : BEDForD, 
1967 ; hamster : Horan \& Bedford, 1972 ; verrat : Holtz \& SMIDT, 1976 ; bélier : FourNiER-DelPeCH et al., 1979). A l'issue du transit, les spermatozoïdes sont stockés dans la queue de l'épididyme d'où sont puisés ceux qui participent à l'éjaculat (SWIERSTRA, 1971).

En insémination artificielle, l'activité copulatoire du mâle, quelle que soit l'espèce, est peu intense du fait que l'éjaculat est fractionné pour inséminer un nombre important de femelles (20 à 30 dans l'espèce porcine). Par contre, elle est beaucoup plus intense en monte naturelle. L'accroissement du nombre d'accouplements peut aboutir à la mobilisation de la totalité des spermatozoïdes utilisables. Fulkerson, SynnotT \& Lindsay (1982) ont montré que, chez le bélier de race Mérinos, lors d'une activité sexuelle intense, le nombre de spermatozoïdes par éjaculat peut devenir inférieur à ce qui est nécessaire pour une fécondation. Ainsi, l'optimum de fécondation ne peut être atteint que chez les femelles s'étant accouplées plus d'une fois (ovins : Schafer \& Mattner, 1966; MatTner \& Braden, 1967 ; bovins : DE BLOCKEY, 1978).

Chez le verrat, bien qu'il soit un gros producteur de spermatozoïdes (SwIERSTRA, 1968, 1973, 1974), les réserves épididymaires sont rapidement utilisées. Le nombre de spermatozoïdes diminue considérablement lors des éjaculations successives et après deux jours de collecte biquotidienne, il décroît de 43 à $17 \times 10^{9}$ par éjaculat (KAPLAN, 1969).

Cependant en liberté, un verrat peut effectuer 4 à 6 éjaculats en 24 heures et, en élevage, il peut être amené à réaliser plusieurs accouplements quotidiens pendant plusieurs jours successifs. Dans l'état actuel de nos connaissances, nous n'avons aucune idée de l'évolution de la production spermatique du verrat dans le cadre d'une monte naturelle intensive.

L'objet de cette étude a été de suivre l'évolution de cette production spermatique chez des verrats soumis à un rythme intensif d'éjaculation, de comparer les caractéristiques des éjaculats obtenus soit par collecte, soit par récupération dans l'utérus après accouplement et, enfin, de mettre en évidence une éventuelle influence sur la fertilité.

\section{Matériel et méthodes}

\section{Verrats}

\section{A. Animaux}

Six verrats ( 2 Large White UPRA et 3 Landrace UPRA) issus de stations de contrôle individuel, âgés de 7 et 8 mois et pesant de 108 à $123 \mathrm{~kg}$ en début d'expérience, ont été entraînés à la collecte pendant une période de 4 semaines avant le début des expériences.

\section{Truies}

Dix-huit truies nullipares Large White, provenant de la Station d'élevage de porcs I.N.R.A. de Rennes-Saint-Gilles, ont été saillies sur cstrus induit pour mesurer les caractéristiques du sperme en accouplement naturel, 24 autres pour apprécier la fertilité. 


\section{B. Modalités d'éjaculation}

1. Collectes de sperme

Lorsque le verrat a chevauché le mannequin, l'éjaculation a été obtenue en exerçant une légère pression à main nue sur l'extrémité du penis. Le sperme a été recueilli dans un flacon préalablement chauffé à $+37^{\circ} \mathrm{C}$ et isolé thermiquement par du coton. Un morceau de gaze faisant office de filtre a permis de séparer la fraction liquide des sécrétions gélatineuses.

\section{Accouplements}

Les accouplements ont eu lieu 24 heures après le début observé des chaleurs. Dans tous les cas, les femelles ont présenté une immobilité immédiate lors de la présentation au mâle. L'observation des accouplements a montré l'existence d'un reflux de sperme non négligeable. Ces pertes ont été récupérées dans un flacon durant l'accouplement.

Afin de mesurer la production de sperme déposé dans l'utérus, nous avons utilisé une méthode analogue à celle décrite par Du Mesnil Du Buisson et DAuzier (1955) dans leur étude de la distribution du sperme dans l'utérus de la truie.

Immédiatement après l'accouplement (délai moyen fin de monte - début d'abattage : $7 \mathrm{mn}$ ), 18 femelles ont été abattues et l'appareil génital prélevé. Puis, celui-ci a été divisé en trois parties : cornes utérines droite et gauche, corps de l'utérus plus vagin. Les deux premières parties ont été perfusées trois fois avec $150 \mathrm{ml}$ de liquide physiologique et la dernière deux fois avec $100 \mathrm{ml}$. Le perfusat a été filtré et recueilli dans une éprouvette avant d'être examiné au microscope pour le comptage des spermatozoïdes.

24 autres femelles ont été abattues 35 jours après l'accouplement; le nombre d'embryons vivants et celui des corps jaunes ont été déterminés.

\section{Paramètres étudiés}

\section{Paramètres quantitatifs}

- Volume de la phase liquide du sperme.

- Volume du matériel gélatineux.

- Concentration.

- Nombre de spermatozoïdes.

Le but de l'expérience étant d'estimer le risque d'une réduction du nombre de spermatozoïdes par éjaculat susceptible d'affecter la fertilité, nous avons relevé les éjaculats possédant moins de $3 \times 10^{9} \mathrm{spz}$, quantité minimale pour obtenir un taux optimal de fécondation en insémination artificielle.

\section{Paramètres qualitatifs}

- P. 100 vivants : il s'agit du pourcentage de spermatozoïdes ayant un mouvement. Il a été noté subjectivement de 5 en 5 . 
- Motilité : les mouvements des spermatozoïdes ont été appréciés selon une échelle notée de 1 (mouvements très faibles) à 5 (progression rapide).

- P. 100 d'anomalies : les anomalies visibles au microscope ont été classées en 5 catégories :

- spermatozoïdes possédant une gouttelette cytoplasmique en position proximale (GP), ou en position distale (GD);

- les anomalies du flagelle (AF);

- les anomalies de la tête (AT);

- les spermatozoïdes sans flagelle (SF).

Lorsque deux anomalies étaient observées simultanément, seule la plus grave était retenue. L'ordre d'importance des anomalies était le suivant : SF $>\mathrm{AT}>\mathrm{AF}>$ GP et GD.

\section{Plan expérimental}

\section{Effet du rythme de collecte sur la production spermatique}

Les verrats ont été soumis à la collecte de sperme pendant 5 jours consécutifs à partir d'un lundi puis ont été au repos sexuel pendant les deux semaines suivantes. Au cours des 5 jours de collecte, un rythme d'éjaculation constant : 1, 2 ou 4 éjaculats par jour a été maintenu pour chaque verrat. Chacun des trois rythmes a été appliqué simultanément. Une permutation du traitement appliqué à chaque verrat a été effectuée toutes les trois semaines.

Les récoltes de sperme ont eu lieu respectivement à $9 \mathrm{~h}, 9 \mathrm{~h}-14 \mathrm{~h}, 9 \mathrm{~h}-11 \mathrm{~h}$ $14 \mathrm{~h}-16 \mathrm{~h}$ pour les rythmes 1,2 et 4 . Les intervalles séparant les éjaculations n'étaient donc constants que dans le cas d'un éjaculat par jour.

\section{Comparaison collecte-accouplement}

Chaque verrat a été soumis alternativement au rythme de 1 ou 4 éjaculats par jour pendant 5 jours après une période de repos sexuel de 15 jours. Cependant, les collectes ont été remplacées par des saillies à trois moments différents : $1^{\text {er }}$ éjaculat

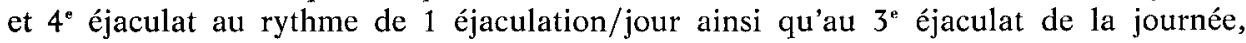
le quatrième jour au rythme de 4 éjaculats/jour.

L'analyse des résultats de concentration en spermatozoïdes, de volume de semence, de production de spermatozoïdes et de motilité a été effectuée à l'aide du test d'analyse de variance. Il en a été de même pour le taux de spermatozoïdes vivant et le taux de spermatozoïdes normaux après transformation des pourcentages en arc $\sin \vee \mathrm{p}$.

\section{Estimation de la fertilité}

Comme ci-dessus, le premier et le quatrième éjaculat au rythme d'une éjaculation par jour ont eu lieu en saillie naturelle et ce, pour six femelles dans chaque cas.

Il en a été de même pour le second éjaculat des $4^{*}$ et $5^{\text {e }}$ journées au rythme de 4 éjaculats par jour. 


\section{Résultats}

\section{A. Effet du rythme de collecte sur la production spermatique des verrats}

Au cours de cette expérience, tous les verrats ont pu être collectés 1 et 2 fois/jour, et 5 animaux sur 6 ont éjaculé régulièrement au rythme le plus intensif.

\section{Paramètres quantitatifs}

\section{a. Volume (fig. 1)}

Le volume moyen de l'éjaculat (phase liquide et gel) ne subit pas de variation significative lorsqu'il est collecté quotidiennement.

A 2 collectes/jour, le volume de la phase liquide et du gel diminue significativement au cours des deux premiers jours. Après quoi, la production se stabilise pour atteindre 50 p. 100 de la valeur du premier éjaculat (effet « jour » : P $<0,001$ ).

A 4 collectes/jour, il est observé deux phénomènes : un effet «jour» et un effet « rythme». Le volume diminue de 55 p. 100 dès le premier jour, puis il apparaît une certaine régularité dans l'évolution du volume des éjaculats au cours des journées suivantes (effet «jour ": $P<0,001$ ). Elle reflète les variations dans les intervalles séparant les éjaculations. Quel que soit le jour de prélèvement, le volume diminue d'une façon significative entre la $1^{\text {re }}$ et la $4^{\circ}$ collecte (effet « rythme »: $P<0,001)$. Enfin, le volume de la phase liquide et celui du gel du premier éjaculat est toujours supérieur à celui du quatrième éjaculat de la veille $(P<0,05): 40$ p. 100 et plus du double respectivement pour la phase liquide et le gel.

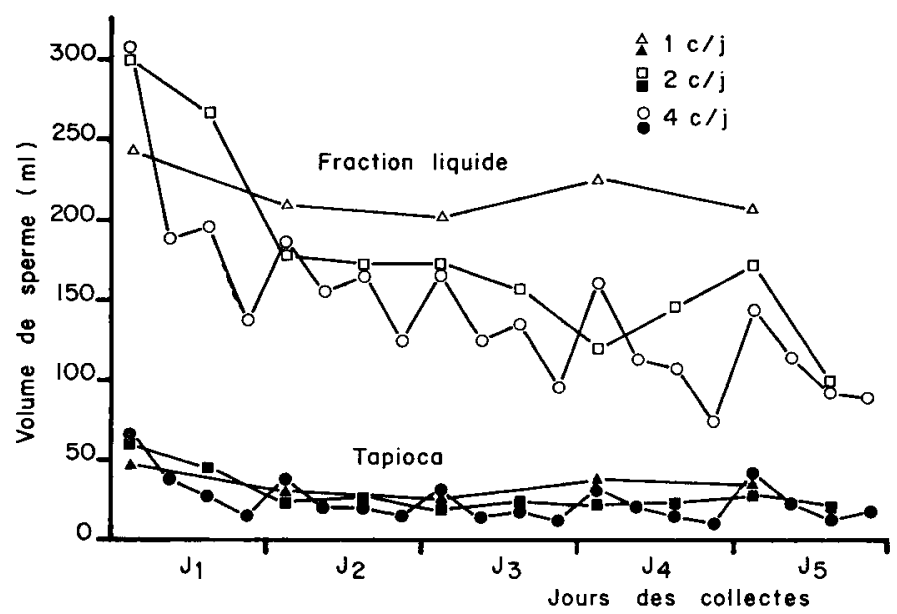

FIG. 1

Evolution du volume de l'éjaculat en fonction du rythme d'éjaculation.

Volume of ejaculate according to the ejaculation rhythm. 


\section{b. Concentration et nombre de spermatozoïdes}

Ces deux paramètres évoluent parallèlement quel que soit le rythme (fig. 2 et 3).

Pour le rythme 1, la concentration et le nombre de spermatozoïdes décroissent régulièrement de $\mathrm{J}_{1}$ à $\mathrm{J}_{5}$ (effet " jour » : $\mathrm{P}<0,05$ ), la concentration diminuant de 68 p. $100\left(163\right.$ vs $\left.52 \times 10^{6} \mathrm{spz} / \mathrm{ml}\right)$ et le nombre de spermatozoïdes de 75 p. 100 $\left(41\right.$ vs $\left.15 \times 10^{9}\right)$.

Pour les rythmes 2 et 4 , l'évolution de la concentration et du nombre de spermatozoïdes par éjaculat est de type hyperbolique (effet «jour»: $\mathrm{P}<0,01$ ). La chute importante du premier jour est suivie d'une diminution plus régulière les jours suivants. Dès $\mathrm{J}_{33}$, la concentration est inférieure à $100 \times 10^{6} \mathrm{spz} / \mathrm{ml}$ et à $50 \times 10^{6}$ $\mathrm{spz} / \mathrm{ml}$ et le nombre de spermatozoïdes par éjaculat n'excède pas $10 \times 10^{\prime \prime} \mathrm{spz}$ et dépasse rarement $5 \times 10^{\prime \prime} \mathrm{spz}$ quand les animaux sont collectés respectivement 2 et 4 fois par jour.

Au cours d'une même journée, la variation dans la durée des intervalles séparant les éjaculations se fait à nouveau sentir : il y a une baisse significative de la concentration et du nombre de spermatozoïdes par éjaculat (effet « rythme» $\mathrm{P}<0,05$ et $\mathrm{P}<0,001$ respectivement pour les rythmes 2 et 4 ). Enfin, dès $\mathrm{J}_{3}$ et $\mathrm{J}_{2 .}$ pour les rythmes de 2 et 4 , le nombre de spermatozoïdes à la première collecte est toujours supérieur à celui qui est obtenu la veille à la dernière collecte $(\mathrm{P}<0,05$ et $\mathrm{P}<0,001$ respectivement pour les rythmes 2 et 4 ). Ce phénomène est également observé à partir de $\mathrm{J}_{::}$pour la concentration au rythme de 2 éjaculations par jour $(\mathrm{P}<0,001)$.

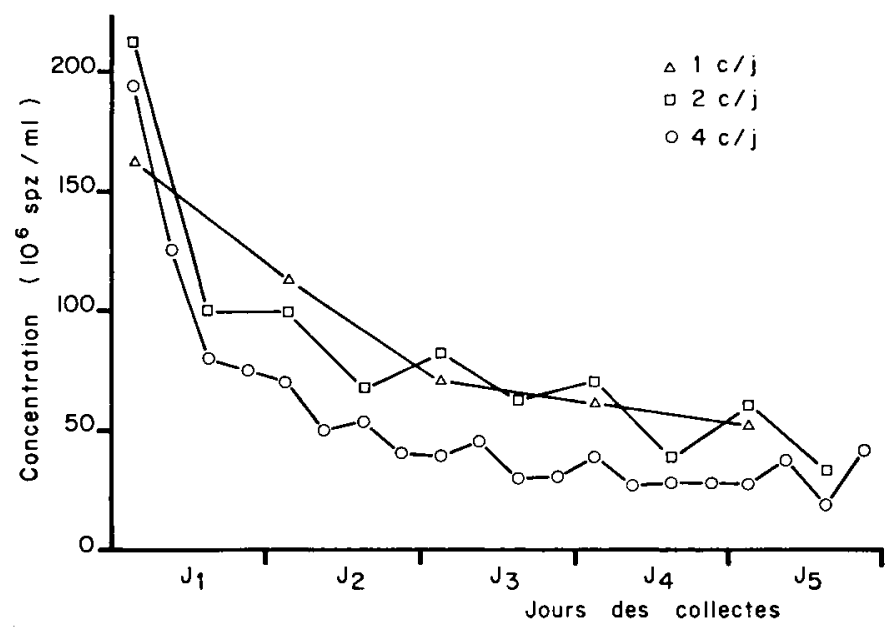

FIG. 2

Evolution de la concentration de la semence par éjaculat en fonction du rythme d'éjaculation. Semen concentration per ejaculate according to the ejaculation rhythm.

L'augmentation de la fréquence des collectes a pour effet de réduire considérablement le nombre de spermatozoïdes par éjaculat. L'étude de la cinétique d'apparition des éjaculats contenant moins de $3 \times 10^{\prime} \mathrm{spz}$ (tabl. 1) montre que ceux-ci 
apparaissent en fin de semaine pour le rythme 2 (10 p. 100 en $\mathrm{J}_{4}, 40$ p. 100 en $\mathrm{J}_{5}$ ), mais s'observent dès $\mathrm{J}_{2}$ pour le rythme 4 et leur nombre augmente régulièrement jusqu'en $\mathrm{I}_{5}\left(20\right.$ p. 100,30 p. 100,45 p. 100 et 60 p. 100 respectivement pour $\mathrm{J}_{2,}, \mathrm{~J}_{3}$, $\mathrm{J}_{4}$ et $\left.\mathrm{J}_{5}\right)$. Cela affecte 10 p. 100 et 31 p. 100 des éjaculats totaux respectivement pour les rythmes de 2 et 4 collectes/jour.

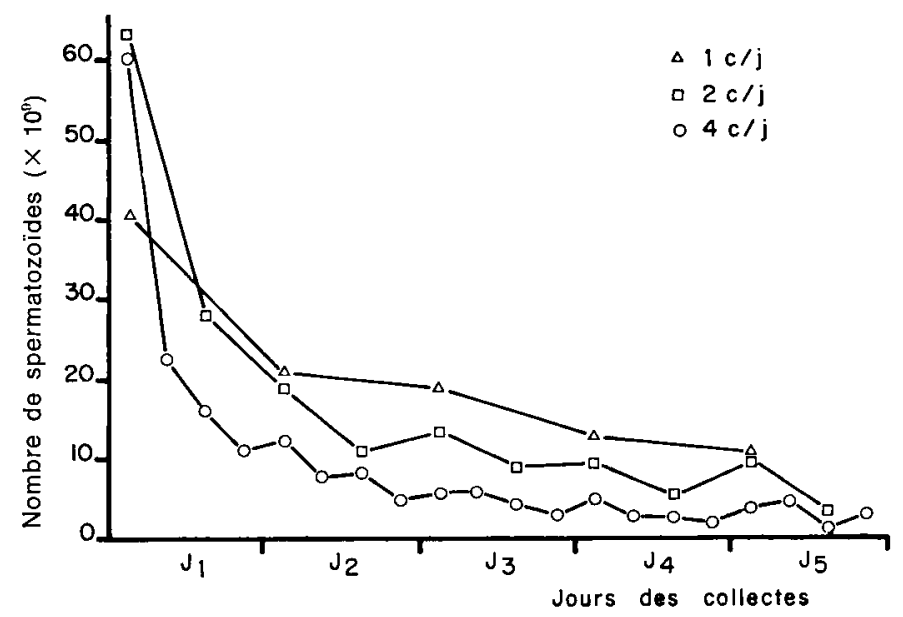

FIG. 3

Evolution du nombre de spermatozoïdes par éjaculat en fonction du rythme d'éjaculation.

Number of spermatozoa per ejaculate according to the ejaculation rhythm.

\section{TABleau 1}

Cinétique d'apparition des éjaculats contenant moins de $3 \times 10^{9}$ spz. (\%).

Kinetics of appearance of ejaculates containing less than $3 \times 10^{9}$ spz. (\%).

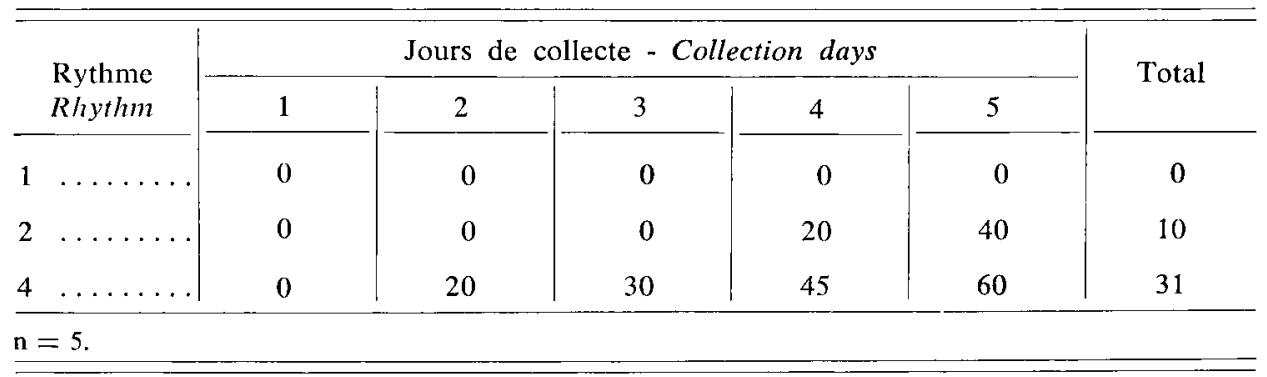

En exprimant la production journalière de spermatozoïdes en pourcentage de la production hebdomadaire (fig. 4), nous constatons que le maximum de spermatozoïdes est éjaculé au premier jour $(38,5$ p. $100 ; 52,7$ p. 100 et 57,7 p. 100 respectivement pour les rythmes 1,2 et 4 ). La production diminue jusqu'en $J_{5}$ atteignant une valeur assez voisine quel que soit le rythme des collectes $(9,2$ p. $100,7,6$ p. 100 et 6,9 p. 100 respectivement pour les rythmes 1,2 et 4 ). 


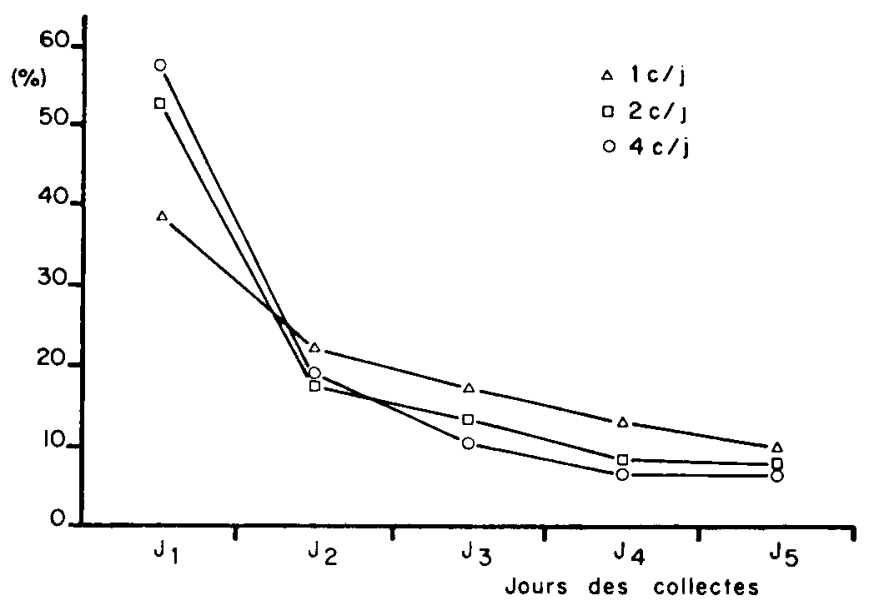

Frg. 4

Evolution de la production journalière de spermatozoïdes en fonction de la production hebdomadaire selon le rythme de récoltes. Daily production of spermatozoa as a function of weekly production according to the collection rhythm.

\section{Paramètres qualitatifs}

\section{a. Pourcentage de spermatozö̈les vivants et motilité}

Une collecte par jour pendant 5 jours n'a aucun effet sur le taux de spermatozoïdes vivants et la motilité. Par contre, pour les rythmes 2 et 4 , le pourcentage de spermatozoïdes vivants augmente significativement de $\mathrm{J}_{1}$ à $\mathrm{J}_{5}(67$ vs $78, \mathrm{P}<0,05$ pour le rythme $2 ; 78$ vs $82, \mathrm{P}<0,01$ pour le rythme 4 ).

La motilité globale, bien que n'évoluant pas au cours de la semaine, présente, pour le rythme 4 , une amélioration entre la première et la dernière collecte de la journée (effet « rythme » $\mathrm{P}<0,05$ ).

\section{b. Morphologie des spermatozoïdes}

La morphologie des spermatozoïdes n'est pas affectée lorsque l'on effectue 1 ou 2 collectes/jour.

Au rythme 4 , le pourcentage de spermatozoïdes normaux diminue significativement (effet « jour » $P<0,001$ ) après avoir atteint une valeur maximale en fin de $I_{1}$ : 65 p. 100 à la première collecte, 76 p. 100 à la quatrième, 55 p. 100 à la vingtième (fig. 5).

Parmi les formes anormales, nous observons une augmentation significative du taux de spermatozoïdes à gouttelette cytoplasmique proximale $(8,5$ vs 14 p. 100 , $\mathrm{P}<0,01)$ et distale $(3,25$ vs 5 p. $100, \mathrm{P}<0,05)$ ainsi que des spermatozoïdes présentant des anomalies de la tête $(1,4$ vs 4,8 p. $100, \mathrm{P}<0,001)$. Il n'y a pas de variation sensible dans le pourcentage d'anomalies de flagelle $(5,5$ p. 100) ou de spermatozoïdes sans flagelle $(0,5$ p. 100). 


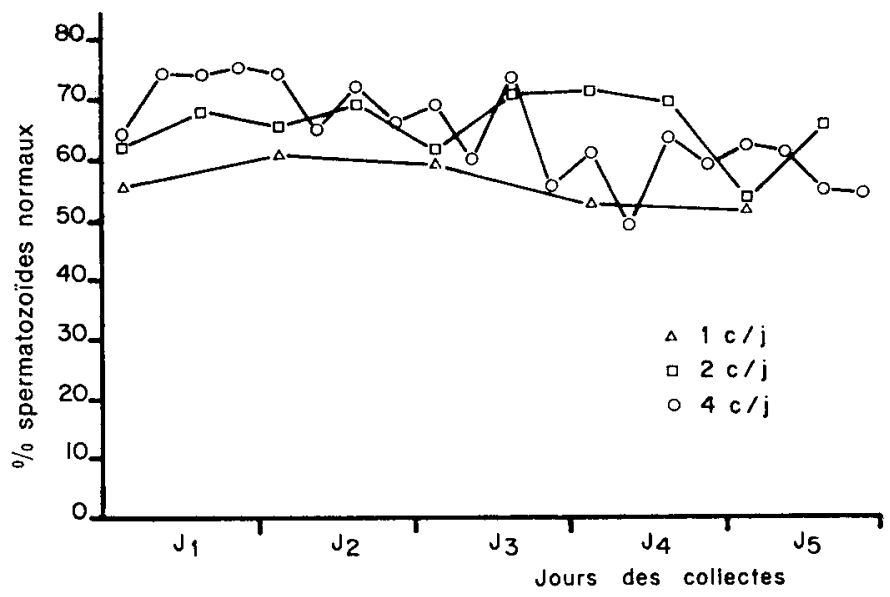

Fig. 5

Evolution du pourcentage de spermatozö̈des normaux par éjaculat en fonction du rythme d'éjaculation.

Percentage of normal spermatozoa per ejaculate according to the ejaculation rhythm.

\section{B. Comparaison collecte-accouplement}

$\mathrm{Au}$ cours de cette expérience, tous les verrats ont pu être collectés 1 fois/jour mais seuls 4 animaux sur 6 ont éjaculé régulièrement au rythme le plus intensif. Durant l'accouplement, nous avons constaté un important reflux de sperme (fig. 6) : sur 16 accouplements, 43,2 p. 100 du volume et 39,2 p. 100 du nombre de spermatozoïdes s'écoulaient à l'extérieur. Lorsque les deux fractions (pertes plus semence déposée dans l'utérus) sont additionnées, il n'apparaît aucune différence significative entre la collecte et l'accouplement dans les paramètres étudiés (volume et nombre de spermatozoïdes).

La répartition des accouplements selon l'importance du reflux (fig. 7) montre deux tendances : zoïdes ;

- accouplement de "bonne qualité » où il y a eu peu de pertes en spermato-

— accouplement de «mauvaise qualité » où les pertes sont importantes.

\section{Estimation de la fertilité}

Le taux de gestation obtenu après accouplement naturel au rythme de 1 éjaculat par jour est conforme aux résultats classiques de la littérature. Par contre, il est très nettement réduit lorsque le mâle a été soumis depuis plusieurs jours à un rythme d'éjaculation intensif. Toutefois, le taux de mortalité embryonnaire n'est pas affecté (tabl. 2). 


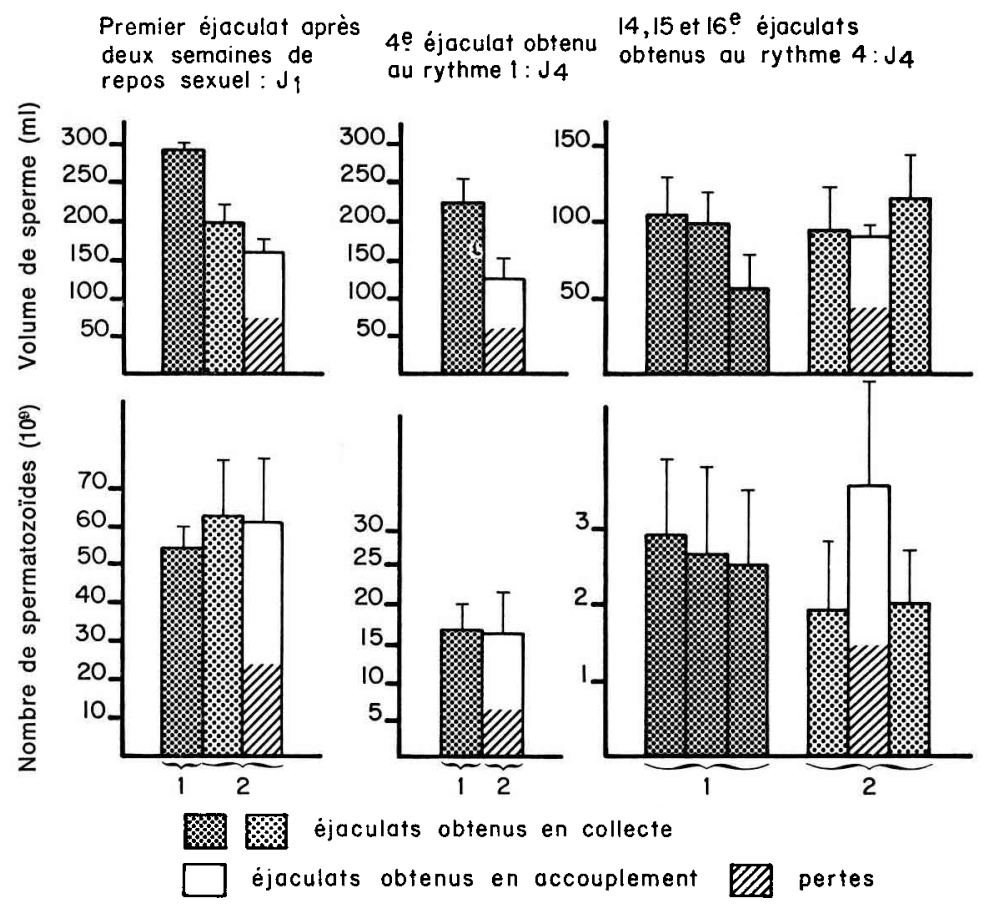

FIG. 6

Effet du mode d'éjaculation:

collecte ou accouplement naturel sur l'évolution du volume de sperme et du nombre de spermatozoïdes.

Effect of the ejaculation mode:

collection or natural mating on the volume of sperm and the number of spermatozoa.

1: $1^{\text {re }}$ phase expérimentale : collecte seulement.

1st experimental step : only semen collection.

$2: 2^{\circ}$ phase expérimentale : alternance de collectes et d'accouplements.

2nd experimental step : semen collection and mating alternately.
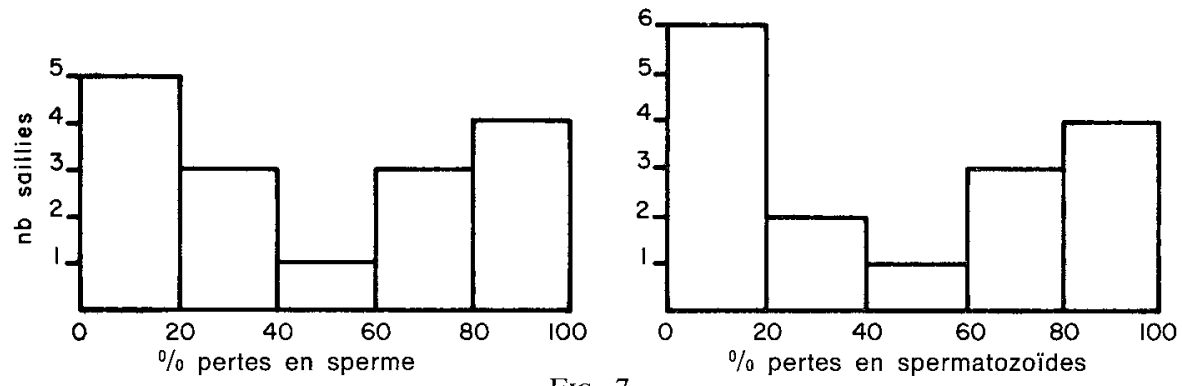

FrG. 7

Répartition des saillies en fonction du pourcentage des pertes en sperme (phase liquide) et en spermatozoïdes.

Distribution of matings according to the percentage of losses of sperm (liquid phase) and spermatozoa. 
TABleau 2

Taux de gestation obtenus après accouplement naturel par des verrats soumis à différents rythmes d'éjaculation.

Gestation rates obtained after natural mating with boars subjected to different ejaculation rhythms.

\begin{tabular}{|c|c|c|c|c|c|c|}
\hline \multirow{3}{*}{$\begin{array}{c}\text { Jour de l'accouplement } \\
\text { Day of mating }\end{array}$} & \multicolumn{6}{|c|}{$\begin{array}{l}\text { Fréquence journalière d'éjaculation } \\
\text { Daily ejaculation frequency }\end{array}$} \\
\hline & \multicolumn{3}{|c|}{1} & \multicolumn{3}{|c|}{4} \\
\hline & 1 & 4 & Total & 4 & 5 & Total \\
\hline $\begin{array}{l}\text { Nombre de truies accouplées } \\
\text { Number of mated sows }\end{array}$ & 6 & 6 & 12 & 6 & 6 & 12 \\
\hline $\begin{array}{l}\text { Taux de gestation }(\%) \ldots \\
\text { Gestation rate }(\%)\end{array}$ & 83,3 & 100,0 & 91,6 & 50,0 & 66,6 & 58,3 \\
\hline $\begin{array}{l}\text { Taux de survie embryonnai- } \\
\text { re }(\%) \ldots \ldots \ldots \ldots \ldots \ldots \\
\text { Embryonic survival rate }(\%)\end{array}$ & 71,6 & 67,3 & 69,2 & 79,5 & 79,1 & 79,2 \\
\hline
\end{tabular}

\section{Discussion}

Cette étude montre que les caractéristiques séminales du verrat sont identiques en accouplement et en collecte.

Les stimulations qui provoquent l'éjaculation sont essentiellement tactiles, que la pression exercée à l'extrémité du pénis provienne de la main de l'expérimentateur ou des tuberosités du col de l'utérus. L'extrapolation des résultats obtenus par collecte est donc possible.

Le volume de sperme, la concentration et le nombre de spermatozoïdes par éjaculat diminuent au cours des éjaculations successives et ce, d'autant plus rapidement que la fréquence des collectes est élevée. En effet, à l'inverse de ce qui est constaté chez les taureaux (Almouist, Amann \& Hale, 1963) ou le bélier (Lino \& BRADEN, 1972 ; Colas et al., 1975), chez le verrat, une éjaculation suffit pour obtenir la plus grande partie des spermatozoïdes disponibles (KAPLAN, 1969 ; SWIERSTRA, 1973). Dès le $3^{\text {e }}$ jour au rythme le plus intensif, le nombre de spermatozoïdes éjaculés au cours de la journée est très proche de la production testiculaire quotidienne (Swierstra, 1971). L'augmentation du rythme d'éjaculation permet ainsi d'obtenir un nombre de spermatozoïdes qui tend vers le nombre effectivement produit par le testicule; cependant, le «gain » ainsi réalisé décroît rapidement lorsque la fréquence des collectes augmente. Ceci confirme la validité pratique du rythme relativement bas choisi pour l'I.A. : de 1 à 3 éjaculations hebdomadaires permettent 
d'obtenir une proportion très élevée de la production spermatique. Il n'en est pas de même en accouplement naturel. Deux éjaculations journalières permettent d'utiliser la quasi totalité des spermatozoïdes disponibles tout en maintenant un nombre par éjaculat suffisant pour obtenir un taux normal de fécondation.

Si un rythme d'une éjaculation par jour n'influence pas la qualité de la semence, il n'en est pas de même pour des rythmes plus intensifs. La composition du sperme obtenue devient telle que la capacité de fécondation consécutive à l'accouplement peut être mise en doute (à 2 collectes par jour, 12 p. 100 des éjaculats contiennent moins de $3 \times 10^{9} \mathrm{spz}$ et, si le rythme est doublé, cela affecte $31 \mathrm{p} .100$ des éjaculats). Or la limite inférieure en insémination artificielle porcine pour maintenir un bon niveau de fertilité est $3 \times 10^{\prime \prime} \mathrm{spz}$ (Signoret, du Mesnil du Buisson \& Bariteau, 1971). Par ailleurs, nous avons été surpris de l'importance des pertes de matériel séminal lors d'un accouplement. Ce phénomène est pratiquement toujours observable (14/16 accouplements). Il n'a été mentionné qu'à l'occasion d'insémination artificielle et n'a que rarement fait l'objet d'une mesure précise. Il ne semble pas exister de relation entre le volume inséminé et le pourcentage de reflux (BAKER, DzIuK \& NorTON, 1968). Ces pertes ne sont pas liées à une perturbation de l'accouplement puisqu'elles ont été observées malgré une immobilisation parfaite de la truie. Elles se produisent avec la même fréquence aux différents rythmes d'éjaculation et ne sont donc pas une conséquence directe de l'importance du volume séminal. Rien ne nous porte à supposer qu'il s'agit d'une situation particulière liée aux conditions de notre expérimentation. Il semble que la possibilité d'un tel phénomène ait été complètement négligée par les chercheurs qui ont étudié la fertilité porcine. Une étude précise sur une grande échelle serait nécessaire pour pouvoir en généraliser les conclusions, lorsqu'il s'agit d'accouplements réalisés à un rythme élevé, ce phénomène revêt une importance particulière étant donné que le nombre de spermatozoïdes se situe à la limite de ce qui est considéré comme nécessaire à une fécondation. Néanmoins, le nombre de spermatozoïdes n'est pas le seul facteur important. Une fréquence élevée d'éjaculations permet tout de même de recueillir un éjaculat volumineux (100-150 ml) qui varie de façon régulière avec l'intervalle séparant deux récoltes (rythme 4). Ainsi, un arrêt des activités sexuelles de $17 \mathrm{~h}$ permet de recueillir un éjaculat dont le volume est supérieur à celui obtenu la veille lors de la dernière récolte. L'existence d'un intervalle de $3 \mathrm{~h}$ au lieu de 2 entre les collectes $2-3$ ne fait pas apparaitre de différence significative entre les volumes des deux éjaculats. Cette reconstitution rapide des réserves des glandes annexes est observée à un niveau moindre en ce qui concerne le nombre des spermatozoïdes. Ainsi des infertilités, liées à une concentration trop faible du sperme peuvent apparaître (BAKEr, Dziuk \& Norton, 1968 ; Stratman \& SELF, 1960).

L'amélioration du pourcentage de spermatozoïdes vivants et de la motilité obtenue avec des rythmes intensifs de collecte peut être expliquée par une durée beaucoup plus faible de stockage des spermatozoïdes dans la queue de l'épididyme.

L'apparition plus fréquente de certaines anomalies dans les éjaculats recueillis 4 fois/jour, telles que les spermatozoïdes à gouttelette cytoplasmique (forme immature), pourrait faire craindre une perturbation des processus de maturation. En effet, il a été montré que la durée de transit diminuait lorsque le rythme des collectes augmentait (bélier : Amir \& Ortavant, 1968 ; coq : DE Reviers, 1973; taureau : OrgebinCRIST, 1962).

Les résultats de fécondation obtenus montrent qu'à un rythme élevé d'éjaculation, 
la fertilité après un seul accouplement peut être affectée. La réduction du nombre de spermatozoïdes mis en place paraît en être la cause.

Dans les conditions d'une activité sexuelle intense, la production spermatique du verrat peut ainsi être le facteur limitant de l'utilisation d'un reproducteur. En pratique, il est donc nécessaire d'envisager une gestion de la production spermatique du mâle, même en accouplement naturel. Par ailleurs, il serait nécessaire de prendre en compte la capacité de production quantitative de spermatozoïdes qui peut être estimée par la taille testiculaire.

\section{Summary \\ Sperm production and fertility in the boar under intensive ejaculation rhythm}

The aim of the study was : 1) to investigate the sperm production of the boar under intensive ejaculation rhythm, 2) to compare in this situation, the number of spermatozoa obtained in semen collection and in natural mating and 3) to study the fertility of gilts mated by boars under different ejaculation frequency. Each boar ejaculated at the rhythm of 1,2 and 4 times per day for 5 days after a period of sexulal rest of 2 weeks. To compare the number of spermatozoa cjaculated in semen collection and in natural mating, oestrus gilts were slaughtered immediately after mating at a given frequency. The fertility of gilts was measured by slaughtering females 35 days after mating.

At the rhythm of 1 ejaculation per day, the total number of spermatozoa per ejaculate decreased linearly from day 1 to 5 an average of $15 \times 10^{9}$; with 2 and 4 ejaculates/day, the decrease was more important on day 1 and reached a plateau at $10 \times 10^{9}$ for 2 ejaculates/day and $5 \times 10^{9}$ for 4 ejaculates/day.

Increasing the ejaculation frequency decreased the number of spermatozoa per ejaculate to a point where it may be under the minimum required for optimal fertilization in artificial insemination, i.e. $3 \times 10^{\text {n }}$; after 5 days this was the case for $12 \mathrm{p} .100$ of ejaculates at $2 /$ day and 31 p. 100 at $4 /$ day.

No differences were observed between the number of spermatozoa ejaculated in natural mating and in semen collection. However, an important and variable fraction of the ejaculate in natural mating flew back from the female's genital tract. Consequently, about 40 p. 100 of the total number of spermatozoa ejaculated in natural mating were not deposited in the female's genital tract. Further, pregnancy rate decreased when the ejaculation frequency increased : 91.6 p. 100 vs 58.3 p. 100 respectively for one and four ejaculates per day.

Consequently, in batch mating of females, resulting for the boars in a high service frequency, and insufficient number of sperm deposited in female's genital tract could reduce the fertility. Thus, the distribution of the spermatozoa output among oestrous females has to be considered in natural mating as well as with artificial insemination.

Key words : Boar, spermatozoa, collection rhythm, natural mating, fertility.

Reçu en fêvrier 1984.

Accepté en mai 1984.

\section{Références bibliographiques}

Almquist J.O., AmanN R.P., Hale E.B., 1963. Sperm output and sexual behaviour of Holstein bulls from 2 to 3 years of age when collected from puberty at high frequency continuously or when given prolonged sexual rest. J. Dairy Sci., 46, 1176. 
Amir D., Ortavant R., 1968. Influence de la fréquence des collectes sur la durée du transit des spermatozoïdes dans le canal épididymaire du bélier. Ann. Biol. Anim. Biochim. Biophys., 8, 195-207.

Baker R.D., Dziuk P.J., Norton H.W., 1968. Effect of volume of semen, number of sperm and drugs on transport of sperm in artificially inseminated gilts. J. Anim. Sci., 27, 88-93.

BEDFORD J.M., 1967. Development of the fertilizing ability of spermatozoa in the epididymis of the rabbit. J. Exp. Zool., 163, 319-330.

BLOCKEY M.A. de B., 1978. The influence of serving capacity of bulls on herd fertility. J. Anim. Sci., 46, 589-595.

Colas G., Personnic D., Courot M., Ortavant R., 1975. Influence du rythme de récolte sur la production de spermatozoïdes chez le jeune bélier Romanov. Ann. Zootech., 24, 189-198.

Fulkerson W.J., SynNotT A.L., LindSAY D.R., 1982. Numbers of spermatozoa required to effect a normal rate of conception in naturally mated Merinos ewes. J. Reprod. Fert., $66,129-132$.

Fournier-Delpech S., Colas G., Courot M., Ortavant R., Brice G., 1979. Epididymal sperm maturation in the ram : motility, fertilizing ability and embryonic survival after uterine artificial insemination in the ewe. Ann. Biol. Anim. Biochim. Biophys., 19, 597-605.

Holtz W., Smidt D., 1976. The fertilizing capacity of epididymal spermatozoa in the pig. J. Reprod. Fert., 46, 227-229.

Horan A.H., Bedford J.M., 1972. Development of the fertilizing ability of spermatozoa in the epididymis of the syrian hamster. J. Reprod. Fert., 30, 417-423.

Kaplan I., 1969. Effect of two collections per day in semen characters in boars. Zivocisna Vyroba, 14, 49-56.

Lino B.F., Braden A.W.H., 1972. The output of spermatozoa in rams. I. - Relationship with testicular output of spermatozoa and the effect of ejaculations. Aust. J. Biol. Sci., 25, 351-358.

Mattner P.E., Braden A.W., 1967. Studies on flock mating of sheep. I. - Mating and behaviour. Aust. J. Exp. Agric. Anim. Husb., 7, 103-109.

du Mesnil du Buisson F., Dauzier L., 1955. La remontée des spermatozoïdes du verrat dans le tractus génital de la truie en cstrus. C.R. Séances de la Société de Biologie, tome CXLIX, 76-79.

Orgebin-Crist M.C., 1962. Recherches expérimentales sur la durée de passage des spermatozoïdes dans l'épididyme de taureau. Ann. Biol. Anim. Biochim. Biophys., 2, 51-108.

DE Reviers M., 1973. Maturation, survie et transport des spermatozoïdes dans les voies génitales mâles des oiseaux. Les Colloques de l'I.N.S.E.R.M., 26, 35-60.

SChafer H., Mattner H.E., 1966. Sexual behaviour of the ram in harem mating in relation to conception rate. Zuchtungskunde, 38, 186-192.

Signoret J.P., du Mesnil du Buisson F., Bariteau F., 1971. L'insémination artificielle porcine. Bull. Tech. Inform. Minist. Agric., 257, 157-162.

Stratman F.W., Self H.L., 1960. Effect of semen volume and number of sperm on fertility and embryo survival in artificially inseminated gilts. J. Anim. Sci., 19, 1081-1088.

SwiERstra E.E., 1968. A comparison of spermatozoa production and spermatozoa output of Yorkshire and Lacombe boars. J. Reprod. Fert., 17, 459-469.

Swierstra E.E., 1971. Sperm production of boars as measured from epididymal sperm reserves and quantitative testicular histology. J. Reprod. Fert., 27, $91-99$.

SwiERstra E.E., 1973. Influence of breed, age and ejaculation frequency on boar semen composition. Can. J. Anim. Sci., 53, 43-53.

SwiERstra E.E., 1974. A comparison of regular ejaculation with sexual rest on semen characteristics and reproductive organ weights in young boars. I. Anim. Sci., 39, $575-581$. 